\title{
Exploring health behaviours: Understanding drinking practice using the lens of practice theory
}

\begin{abstract}
Research suggests that there is no safe amount of alcohol but despite this alcohol consumption remains an important part of many [young] people's lives. Viewed as an inherently social activity drinking alcohol provides an opportunity for socialising and connecting with friends. This study is one of the first to draw on practice theory to explore one type of intoxicated drinking occasion engaged in by young people; framed in this paper as a 'proper night out'. This article argues that this hybrid entity is made up of a series of interconnected social practices that have come and now hang together to serve to normalise and routinize intoxicated drinking occasions. The operationalisation of practice theory has enabled an indepth exploration of intoxicated drinking and provides the potential for new ways of intervening in harmful drinking practices by refocusing attention away from individual level decision making to drinking practices.
\end{abstract}

Authors; Dr Kath Hennell, Liverpool Hope University (UK), Professor Maria Piacentini, Lancaster University (UK) and Dr Mark Limmer, Lancaster University (UK) 


\section{Introduction}

Non-communicable diseases such as cancer and heart disease associated with individual lifestyles and health related behaviours, such as smoking, alcohol consumption, lack of physical exercise and poor diet (Holman and Borgstrom, 2016; Blue et al., 2016) are a leading cause of mortality and disability worldwide (WHO, 2012). Alcohol consumption in particular has been associated with a considerable social, economic and disease burden in Western societies (WHO, 2018). While alcohol data suggests a complex consumption picture, especially among 16-24-year-olds, intoxicated drinking remains a concern in countries where alcohol consumption is high such as European countries including the UK, Australia, Canada and New Zealand (WHO, 2018). Measham and Brain's (2005) concept of a 'culture of intoxication' is useful in understanding the determinedness of drunkenness where the aim of a night out is to become intoxicated (Fry, 2011) and this is not only accepted, but also expected and normalised within many young people's social lives (Mackiewicz, 2015). However, it is also a space where gender and class distinctions intersect to shape notions of suitable and acceptable performance of intoxication (Mackiewicz, 2015; Waitt and De Jong, 2014).

Interventions to prevent harmful health behaviours including the consumption of alcohol have frequently been reduced to the individual level (Kelly and Barker, 2016; Blue et al., 2016; Maller, 2015). Despite there being little evidence to 
suggest that these such interventions have made large scale health gains (Martin, Nelson and Lynch, 2013; Meier et al., 2018). Internationally, public health groups, sociologists and anthropologists have repeatedly called for a broader understanding of those harmful health behaviours surrounding alcohol consumption including heavy episodic drinking, consumption above low risk guidelines and daily drinking (Thom et al., 2016; Fraser, 2018; Maller, 2015). Within this context there is a growing recognition that a practice framework can contribute to this wider understanding (Maller, 2015).

Practice theory encompasses a range of theorists who offer an alternative way of understanding human action, shifting the focus away from the individual on to the practice itself (Shove et al., 2012; Reckwitz, 2002; Hargreaves, 2011). A practice in this sense is an activity, a 'doing' or an entity (Schatzki, 2012) which emerges from the social organisation of everyday life (Reckwitz, 2002). Schatzki (2012: p14) describes a practice as an "open ended, spatially-temporally dispersed nexus of doings and sayings". Individuals are conceptualised as carriers of the practice who facilitate the existence of practices through their performances in their everyday lives (Shove et al., 2012; Warde, 2005).

Practice theories have been used primarily in the development of an international body of work on sustainability that have challenged the dominant discourses around environmental and climate change policy (Hargreaves, 2011; GramHanssen, 2011). More recently, practice scholars have applied this theoretical 
lens to a wide range of activities related to health globally such as smoking, snacking and cycling (Spotswood et al., 2015; Twine, 2015; Blue et al., 2016). Despite this, there are few empirical studies researching alcohol consumption using a practice theory framework. Notable exceptions are Ally et al. (2016), Supski et al. (2017) and Maclean et al. (2018). These studies build on previous research that moves away from privileging individual behaviour as a conduit for change to a practice theory approach that encourages a more sociological understanding of health behaviours by exploring drinking as a social practice among university students in Australia (Supski et al., 2017) and the drinking practices of same-sex attracted women (Maclean et al. 2018). Ally et al.'s (2016) study of different typologies of alcohol consumption, recognised that more research is needed into drinking occasions, particularly those that are constitutive of mixed drinking locations. Furthermore, the wider literature on alcohol has in the main tended to neglect the relationship between drinking and other social practices (Meier et al., 2018; Duff, 2018).

This paper responds to the work of Meier et al. (2018) who call for a new approach to alcohol epidemiology and intervention research. Specifically, they assert that research that explores how drinking practices emerge, persist and decay over time and how they relate to other practices within daily life will offer new insights into drinking trends, patterns and alcohol related activity and potentially how public health interventions can address them (Meier et al., 2018). 
Whilst Meier et al. (2018) call for more quantitative practice based research studies, Fraser (2018) argues that research needs to make use of both quantitative and qualitative methodologies if we are to better understand the various elements that come together to (re)produce and sustain drinking practices.

This study is the first to adopt a social practice lens and a qualitative methodology to understanding the intoxicated drinking practices of young people outside of a university environment. This study aims to understand intoxicated drinking occasions within a practice framework; to develop understandings of and insights into the routinised actions of young people in relation to these practices; and to develop understandings of the relationships between alcohol consumption and other social practices that interconnect to the drinking occasion.

\section{Social practice theory}

This study follows the lead of Spotswood et al. (2015) and Supski et al. (2017) in using the three elements model of social practice theory as conceptualised by Shove et al. (2012). According to this framework, individuals do what they do because of the relationship and combination of three interdependent social practice elements: materials (stuff, things, technologies and tangible, physical entities), meanings (common understandings, symbolic meaning, ideas and aspirations) and competences (skills, know-how and techniques) (Shove et al., 2012: p14). In addition, I follow the example of Meier et al. (2018) who argue that the inclusion of temporal rhythms, timings and duration of the activity, is 
relevant for any study of drinking practice. This approach enables an exploration of the inter-relationship between drinking practices and other practices including those in competition such as parenting and work which compete for time and resources. In addition, there are important aspects to how practices (inter)connect to other practices as a bundles arrangement 'based on co-location and coexistence' (Shove et al., 2012: p81) or which are 'materially interwoven' (Schatzki et al., 2001: p3). For example, drinking alcohol can connect with celebrating, leaving work and eating. The interconnections between practices can result in the emergence of complexes of practices (tighter arrangements based on 'sequence, synchronisation or proximity') or new hybrid entities (Shove et al., 2012: p87). Understanding that drinking occasions are interconnected with other practices will enable a more in-depth understanding of intoxicated alcohol consumption. (Maller, 2015).

\section{Data and methods}

The study was an in-depth qualitative project conducted over 14-months. Data from young people's narrative accounts given in group interviews and their social media content relating to both alcohol consumption and (inter)connected practices were collected and analysed. This approach builds on the precedents of other social practice research studies that have also used a qualitative methodology (Nairn and Spotswood, 2015; Spotswood et al., 2015; Warde, 2005). There has been considerable debate about the usefulness or otherwise of 
conventional qualitative social science methods, such as interviews and focus groups for studying practice (Martens et al., 2014). A frequent criticism levelled at the effectiveness of these methods within a social practice framework is that embodied knowledge is thought to not easily lend itself to verbal communication (Martens et al., 2014; Nairn and Spotswood, 2015). Jenkins (1994) argues that the researcher is required to get around the 'official account' given by the research participant as the account omits the obvious, for example, gender performance. In this sense the account itself represents a performance, in much the same way as the practice does. Despite the criticism, prominent social practice theorists such as Blue et al., (2014), Hargreaves (2011) and Gram-Hanssen (2015) have argued that qualitative methods should have a place within social practice theory. Indeed, a robust defence of interviews within practice-based research has been articulated by Spotswood et al. (2015), Hitchings (2012) and Martens (2012) who reason that the researcher can and does get some idea of practical activity through linguistic articulation. Martens (2012) suggests that verbal insight could be further enhanced by a research context that enables additional insight into the practice; for example, by combining observation and talk. This study therefore uses a qualitative methodology which combines talk based methods and observation of social media content.

The study draws on data from three mixed gender friendship groups $(n=23)$ recruited from low income neighbourhoods in North West England. Group details 
are provided in table 1. A snowballing approach to recruitment was adopted. Previously known contacts acted as gatekeepers to wider networks of contacts, and from these, three friendship groups of young people, previously unknown to the researcher, aged between 16 and 21 years of age were recruited. Each group participated in three or four interviews, approximately three to four months apart. Group interviews lasted from 60-75 minutes and were semi-structured with discussion of relevant practices illuminated by social media content. Interviews were conducted in places convenient to the young people, for example, private space in college or workplace and were digitally recorded. Transcription followed an orthographic approach with minimal cleaning (Braun and Clarke, 2013).

Group interviews offered this study the potential to uncover particular ideas of shared routines and cultural conventions, and identify challenges to these social norms through inconsistencies highlighted between participants (Nairn and Spotswood, 2015). In addition, they offered the potential to highlight the different performances of a practice, identify the materiality, meanings, competences and temporal rhythms of the practice, and the connectivity between these and other practices.

Participant's social media content from Twitter, Facebook and Instagram relating to drinking practice, competing practices and interconnecting practices was collected. Content including visual images, hashtags, microblogs, on-line conversations and memes was collected on a monthly basis. Facebook content 
was collected via each participant's timeline to circumvent Facebook algorithms (the set of technical elements, intricate logic, and usage analytics that delivers content to users) and editing and all social media data was captured in PDF format. Each PDF was then edited for material that was deemed appropriate such as content associated with alcohol, drinking practices, going out practices and competing practices.

\begin{tabular}{|l|l|l|}
\hline Group type & Participants & Ages \\
\hline Apprentices & $2 \mathrm{M}, 2 \mathrm{~F}(4)$ & $19-22$ years \\
\hline Post 16 college students & $2 \mathrm{M}, 9 \mathrm{~F}(11)$ & $16-18$ years \\
\hline Post 16 college students & $3 \mathrm{M}, 5 \mathrm{~F}(8)$ & $18-21$ years \\
\hline
\end{tabular}

Table 1: Participant demographics

In order to interpret these different and diverse forms of data and operationalise social practice theory, interpretation of the data involved the integration of all source data and analysis with the combined data set following the example of Nairn and Spotswood (2015). A hierarchal code labelling frame using the affordances of NVivo software was produced. This combined practice theory conceptualisations with methodological insights from both Braun and Clarke's (2006) thematic analysis and Banks' (2008) qualitative visual data analysis who proposes the notion of an internal narrative which focuses on content and an external narrative which refers to the context in which the image is observed. Hashtags, microblogs and on-line conversations were constituted as the 'sayings' 
of the practice and were explored as the voices of the participants but also in relation to the 'doings' of the practice (Schatzki, 2016).

Additional codes were generated from emergent themes arising from the data resulting in a thematic map that was both theoretically and data driven. The process involved an ongoing analysis of data and a refining of themes to develop an in-depth understanding of and insight into the essence of these themes and a deeper insight (Nairn and Spotswood, 2015).

In line with SRA (2003) ethical guidelines each participant gave informed consent at the start of the study and re-consented at each interview as part of an ongoing process of revisiting and contextualising consent. Pseudonyms were ascribed to each participant to protect their identities. The study was subject to institutional ethical regulation and scrutiny.

It is important to note that the small number of friendship groups in the study makes these findings less generalisable. However, the intention was to draw upon a highly localised and small sample over an extended period, which generated considerable amounts of data. In addition, it should be recognised that young people participate in different types of alcohol consumption practices and analysis of these other types of drinking practice combined with a tracking of their practice trajectories would further broaden understandings of young people's alcohol consumption.

\section{Findings}


The study findings show that participants were involved in various drinking occasions including consuming alcohol with a meal or with a family and friends. However, there was one specific drinking occasion that they engaged in (to a greater or lesser degree) that included the type of determined drunkenness that is constitutive of the 'culture of intoxication' (Measham and Brain, 2005). This drinking event was described as a 'proper night out' (PNO). Findings are used here to develop an in-depth understanding of the arrangement of practices that hang together to form a PNO and the routinised actions of young people in relation to intoxicated alcohol consumption. The aim is not to provide an objective, factual description of this arrangement bundle, but rather to enable an in-depth understanding of the bundle and its constitutive practices and elements to be developed.

The PNO is important because firstly, young people's nights out are considered to be a high-risk drinking occasion (Ally et al., 2016, Measham and Brain, 2005). And secondly, because the PNO is an event that captures both pre-drinking (generally in someone's home) and commercial public spaces and this type of drinking in a mix of public and private locations is associated with high alcohol consumption levels (Ally et al., 2016; McCreanor et al., 2015).

The phrase the PNO was used by three of the young women; who in so doing framed the occasion as something more than just going 'out' and 'getting drunk'. The word 'proper' is used as an intensifier to emphasise the 'night out' and 
distinguish it from other types of occasion. By focusing on a PNO, the unit of analysis is sufficiently narrow to enable an in-depth investigation, but broad enough to enable an exploration of the complexities of determined drunkenness (Fry, 2011) and inter-related practices. A PNO is therefore situated as an alcohol consumption practice bundle arrangement that is located within the social, temporal, economic, gendered and cultural organisation of the everyday.

There was no consensus from the participants on the frequency of a PNO although it was sometimes linked to a celebration such as a birthday or a workplace celebration. Participants associated a PNO usually with a Friday and/or a Saturday and for some it was a weekly occurrence and for others, more infrequent, sometimes once a month or even every other month.

Participant's involvement in the PNO practice arrangement was constrained by their limited time resources and bounded by the impossibility of being involved in more than one competing practice at any given time (Shove et al., 2012). Although there was no specific duration identified by participants, there was a general consensus that the 'going out' element was carried out over a number of hours frequently into the early hours of the following morning, or as an all-day event. Andy, for example, referred to getting the first train home in the morning after a night out and in a similar vein, Claire described getting a train into the night-time environment just before midnight. Mark recounted a story of walking home the following morning in the previous night's clothes, referring to this as 
the "walk of shame". As a result of this lengthy duration, competition from other practices for time resources affected the timing and frequency of the young people's participation (Shove et al., 2012). For example, only two groups gave an example of participating in the PNO on a weekday evening. Andy talked about a party he had attended on a Thursday night, however this was when he had finished school and there was no practice competing for time resources the following day. Similarly, Mark, Leah and Emily described participating in a PNO during the week shortly after starting their apprenticeship scheme. However, they were in work the next morning and perceived it to have had an impact on their work performance. As they became accustomed to being in paid employment, they explained that their participation in the PNO during the week lessened as the competing practice of work meant that it was no longer viable. The lengthy duration indicates that a considerable temporal resource needed to be invested in the PNO and the competition from other practices such as familial and work practices for this temporal resource affected both frequency and the time that young people are able to participate in the PNO.

\section{The bundle arrangement of a PNO}

Analysis of the data reveals that the PNO followed a similar configuration for all participants and six constitutive practices were identified; planning, getting ready, pre-drinking, going out, getting home and storytelling. Each practice could 
also be independent from the PNO or hang together in a loose collection with other practices to form other practice bundles. For example, other drinking occasions may be planned and arrangements made prior to participation. However, these practices were integral to the bundle arrangement and were performed by all carriers to a greater or lesser extent.

Firstly, participants engaged in planning and arranging the event; typically preceding the PNO occasion. On the day, preparation of self was integral to getting ready. Pre-drinking either followed getting ready or combined with it. These were then followed by going out; either in the night-time economy or to a party. The final practices within the bundle were getting home and storytelling; where highlights of the night were shared with a wider audience.

Leah neatly summarises four of the practices, getting ready, pre-drinking, going out and getting home; "So we would get dressed have drink at ours first, have our music on, get a taxi. We would do the rounds in town and then we would probably go back to mine.". Following the final practice of storytelling, the planning of the next PNO commences. 


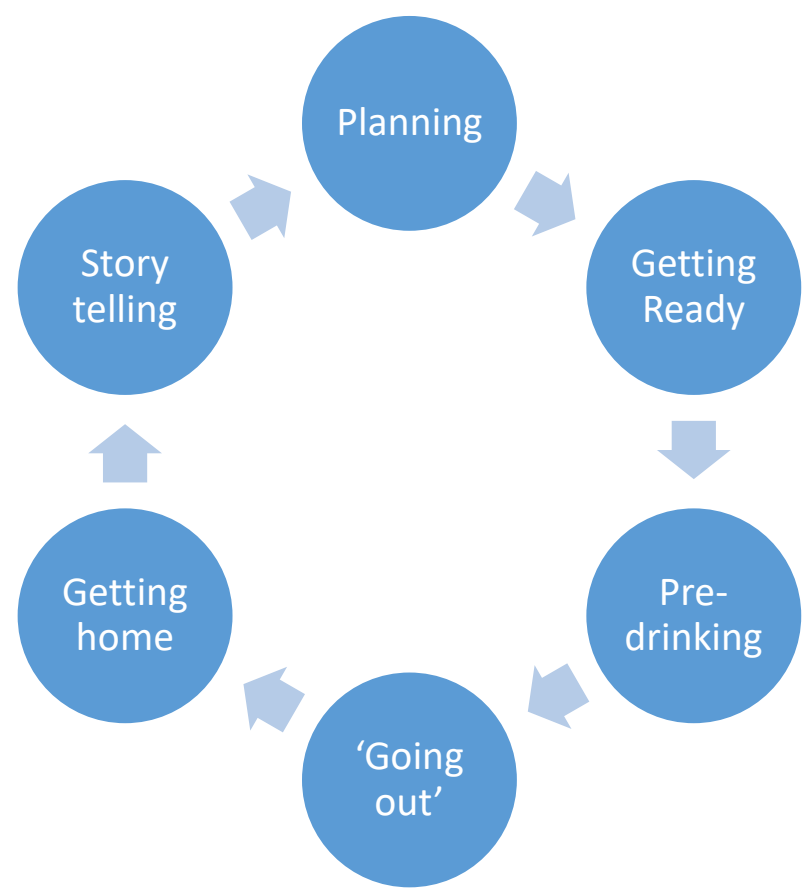

Diagram 1: Practices of a PNO

\section{Planning}

Planning and making any necessary arrangements was a critical part of the practice and took place over the preceding day(s) or weeks depending on the occasion. The more special the occasion was deemed to be, the longer the planning time, as illustrated by Amy: “.. but when you are like planning like a birthday then it's like a month in advance".

Arrangements could range from the elaborate, for example Mark's $21^{\text {st }}$ birthday event which involved booking a room in a hotel, to the more 'usual' arrangements that a friendship group made for a PNO. Liam explains ".. basically we had a surprise birthday and it was massive party for like my best mate. So obviously you would organise that differently". A PNO required planning to make these nights extra special and differentiate them from other types of nights out. 
Examples included planning nights away from home or the wearing of fancy dress. James explained, "going out is tactical-you have to plan it". Being tactical in the planning was also a way of regulating risk. For example, planning transport for getting home safely. The specifics of these arrangements differed from group to group but each of the participating groups had a strategy for getting home safely from a PNO.

\section{Getting ready}

Group messaging using social media platforms was routinely used particularly by female participants discussing potential outfits, hairstyles and overall appearance, allowing them to comment on each other's possible assemblages. The young women drew on a discourse of an appropriate look for the PNO. This look was highly gendered, highly sexual and feminine in appearance with a fully made up face, hair stylised and the wearing of heels which was considered an absolute necessity. Charlotte explains that "If we are going into town it would be like heels, full face of make-up, dress and hair completely like curled - everything!"

The sharing and commenting by group members enabled each potential outfit to be appraised and the 'right' look endorsed. This was carefully managed as illustrated by Amy who suggests that offering a few options for comment reduces the risk of wearing an unsuitable outfit: "If normally a suggestion is made about what you are going to wear there is like normally a few options [shared on social media] so you can go for the one that's, like, is the least like you know 'I don't 
like that' type of thing." These images conveyed knowledge about the practice materials such as getting the right look which was then also linked to the practice performance by understandings of what look was acceptable and what was not. The finished 'look', would then be shared online with their social networks, usually with a visual image. The virtual social network was then invited to appraise the look by 'liking' the image and to make positive comments.

This practice could, however, be constrained by competing practices. In the example below Claire and Becky both work on a Saturday day, which limits the time available for getting ready. Therefore, the two young women meet up with their respective boyfriends whilst getting ready and then meet for pre-drinks at the local pub.

Researcher: Do you do pre-drinking?

Claire: We always have a certain pub we go to before we get the last train.

Researcher: So you wouldn't get together before you go to the pub?

Claire: $\quad$ No cos we are at work.

Researcher: So you go straight from work?

Claire: $\quad$ No I get ready - get a shower and stuff.

Researcher: And you don't meet up with anybody else before the pub? 
Claire: $\quad$ Yes, I get ready and then we meet.

Researcher: So all your pre-drinking is in the pub? You wouldn't have a drink whilst you were getting ready?

Becky: $\quad \quad \quad$ Yeh I'd have a drink when I am getting ready. But that's just like a little drink whilst you are getting ready.

Researcher: Ok so a little drink whilst you are getting ready. And then that's just you on your own getting ready?

Becky: $\quad$ No there's my boyfriend. He usually comes round.

In this example Getting ready formed part of the embodied practice competences and it did not easily lend itself to explanation (Martens et al., 2014). Demonstrated by the eventual acknowledgement that boyfriends were also present and that alcohol consumption is embedded within their practice. This contrasts with the young men in the study who narrated a part in the getting ready practice only when they were participating in the practice in the company of female friends. Mark for example explained that "either I'll go to Emily's to get ready or she'll come to mine and we'll get ready."

The young men who participated in the PNO in all male groups did not narrate stories of getting ready together as a group of friends in the same way as was portrayed by all female groups or mixed groups. However, analysis of their social media suggests they did participate in the practice of getting ready as these 
images were of well-groomed, similarly styled young men indicating some degree of co-operation or a tacit understanding of what to wear and getting the 'right look'.

The lack of stories relating to this practice by the male participants suggest that it is the planning practice where decisions are made on what to wear and getting ready is solitary except with female partners or friends. It is not until the next stage that the young men interact with their male friends. This illustrates how the performance of this practice is gendered with the all-male groups positioning their masculinities in relation to more conventional masculine traits such as independence and self-reliance to justify their male grooming (Dempster, 2011).

\section{Pre-drinking}

Pre-drinking, enables the social group to come together, bond and cement their friendships and provides an opportunity to consume alcohol cheaply, in order to reach the desired intoxication levels (McCreanor et al., 2015; Forsyth, 2010). In contrast to the findings in previous literature on pre-drinking (McCreanor et al., 2015; Forsyth, 2010), the research participants undertook this practice in both public and private settings. However, public spaces for pre-drinking were away from the main night-time environment or party venue frequented in the going out stage, although they were often en route. For example, a local pub or bar close to a train station or on the edges of the city centre. 
Narrations of Pre-drinking suggests that this practice was gendered and performed differently by the young men and women in the study; the young women talked of frequently drinking together in small groups during the getting ready stage and their visual images on social media also demonstrated this point. Engaging in pre-drinking in this manner enabled the young women to concentrate on getting ready by simultaneously providing access to the essential material elements; power sockets for hairdryers and straighteners, mirrors, good lighting, makeup, fake tan and so forth. However pre-drinking was not solely the preserve of young women; all-male friendship groups were also involved in pre-drinking as the following discussion demonstrates.

Researcher: So Andy do the lads get together before they go out?

Andy: $\quad$ Yes at someone's house. For pre-drinks.

Researcher: So does everybody bring something? How does it work?

Andy: $\quad$ It depends how many there are. If there are only a few then we might just get a litre of vodka between us all just for a predrink - between four of us.

However, the young men did not (usually) combine the social practice of predrinking with getting ready; rather these were narrated as two distinct practices. Pre-drinking was performative in nature, giving them the opportunity to 'catchup' with friends and to consume sufficient amounts of alcohol so as to enable them to reach acceptable intoxication levels. The embodied experience of 
intoxication was constitutive of the PNO, however, this intoxication experience was highly gendered in that different practice performances were enacted by the young men and women. For example, men enacted an embodied masculinity through an intoxicated loss of bodily control (Thurnell-Read, 2013) which was narrated as less controlled and less constrained than traditional masculinities associated with heavy drinking (Campbell, 2000). In contrast, drinking excessively has commonly not been regarded as performing acceptable femininity (Bailey, Griffin and Shankar, 2015) and the young women enacted a performance of a more controlled loss of control (Measham, 2006). For example, Phoebe constructed a narrative that was constitutive of controlled drinking, by drinking within her intoxication limits and describing her desired intoxication level as "tipsy". In her storytelling Phoebe is rehearsing a respectable femininity that distances her intoxicated self from the working class binge drinker depicted in popular discourses (Haydock, 2014).

\section{Going out}

This practice together with getting home, frequently formed the most visible and public part of the PNO (Niland et al., 2013; Chatterton and Hollands, 2003; Roberts, 2013). Maintaining intoxication at an acceptable form and level (thus representing acceptable practice performance) formed a part of the practice know-how. Going out was typically performed differently in different locations depending on whether the participant was under the legal drinking age or of legal 
drinking age. The younger participants performed going out in private party venues and the over 18 years' age group in the bars and clubs within the nighttime environment. Liam, age 16 stated, "I just like house parties". In the main this was because of issues associated with providing photo identification. The issue was demonstrated by Olivia who no longer goes out into the night-time economy after being refused entrance to a licensed venue.

Olivia: I used to go to xxx all the time but I am only 17 and I started getting KBd [rejected] and I was like oh no tara. And just didn't go again

The importance of the materiality of the practice is highlighted by the participant's stated preferences for specific bars and clubs. This depended on the type of music played, perceptions of the atmosphere and the perceived likelihood of knowing other bar/club goers. A number of bars would be frequented during this stage with participants expressing a dislike for staying in one place, although the order for visiting was not usually decided beforehand. Moving from one bar to another was part of the routine of the evening while staying in one venue was considered 'boring' and movement created a sense of excitement to both the practice and to the storytelling with the different venues visited shared on social media.

Each member of the group would be familiar with the preferences of their group and typically, these were the bars that would be visited. For example, in the 
following extract Lauren and James discuss how their preferred venues have a client group that knows and is familiar with each other.

Lauren: Everyone knows each other anyway it's like a huge family in xxx.

James: I think that's why a lot of people are attracted to the same bars cos you know everyone there... And like I know the vast majority of people in xxx. So it's all really familiar.

New bars would be visited when participants were enticed by special offers or persuasive promotions. These bars could then be added to the preferred list of bars or alternatively not visited again. Knowledge about which bars were 'good' was passed on from older siblings or friends as Harry explains.

Harry: We went there because it was the most places people went and kept on talking about. And that's when we thought we might as well try that.

This finding illuminates how going to certain bars or house parties and not others created both group identities and group membership. This was further shared with the wider community through individual participant's social media posts enabling valued drinking spaces to be shared with a wider group. For instance:

Let's get this party started! xxx - at [venue] Street

Mmmmmmm Mark xxx - at [venue] 
Paradoxically, there was a difference between the narratives shared in the group interviews of bars frequented and the types of venue appearing on the participants' social media. For example, some participants talked about frequenting bars that were shabby but known to serve cheaper drinks such as chains and bars on the fringes of the town or city centre. These types of bars were not visible on social media as they were viewed by participants as being less desirable and as such unlikely to fit with middle class notions of taste and aesthetic preference, and therefore unlikely to lead to the acquisition of status through shares, retweets and likes.

\section{Getting home}

Getting home was an important aspect of the PNO. It featured heavily in discussions during the planning practice. The young women worried about their vulnerability at this time and had strategies in place to ensure they got home or to a place of safety. Although the young men did not discuss getting home in terms of their own safety their planning narrations included a safety discourse around ensuring female friends got home safely. Similarly, to the young women this practice featured strongly in the planning by the young men although this practice was clearly gendered. The young men's enactment of a protective masculinity and the young women concerns about getting home safely are rooted in the gendered fear of sexual violence and culturally embedded safety concerns. The getting home strategy was also classed and dependent on how far away the young 
people lived from the drinking location and the availability of financial resources. Examples of getting home in financially constrained circumstances included; getting a lift, getting the first train home in the morning, or asking another friend to collect them.

\section{Storytelling}

Previous studies have demonstrated the importance of storytelling in the post consumption phase as a way of reinterpreting and reconstructing the drinking experience (Fry, 2011; Niland et al., 2013). This practice allows the embodied drinking experience to be enhanced, repeated and solidified (Fry, 2011). The use of technological items, specifically mobile phones and their affordances of social media have been seen to be an important material element of some of the previous practices but is particularly pertinent to the practice of storytelling as social media was used to tell, share and re-tell accounts of drinking related events.

Narrations of the PNO on social media were often focused on the telling of humorous experiences of intoxication, or associated with specific alcohol brands, attendance at events, drinking locations and contexts, often accompanied by images. This content served to consolidate and accumulate social status and approval through its recirculation, 'likes', comments and retweets.

These narrations provided a way of conforming to social group values, whilst distinguishing the self from others, for example, other age groups, the opposite sex, consumers of particular alcoholic drinks and patrons of particular bars. The 
affordances of the digital environment enabled young people to act out individual and group identities to different social media audiences, posting statuses, tweeting and posting photographs of their PNO. The throwback functionality of social media enabled nostalgic content in the form of previous drinking occasions to be re-shared further extending these occasions. Social media content is then recirculated and approved by their virtual social networks, for example, on Snapchat, accounts of the PNO were shared between an intimate and close-knit peer group whilst Facebook and Twitter content were used to post accounts to wider social networks of family, friends and colleagues. It can, therefore, be suggested that there are a number of different audiences for the virtual performance of the PNO: the friendship group themselves who have shared the experience, the different social media users who access different virtual performances, and in this instance the researcher.

It is interesting to note that the social media content was shared with a number of different social networks not just peer networks as identified in a study by Atkinson et al. (2015). In this way the performance of the practice was multilayered in nature and differ according to the varied audiences. The following social media posts show post-drinking accounts of intoxication:

Am I arsed no! \#foreveramess

Hangover food FTW! xxx 
Dog rough $x x x$

If there's ever a reason I shouldn't drink this is it \#absolutestate

Every time..... you can guarantee

The retelling of stories, either virtually or otherwise, served to cement the friendship, binding the group together by providing a shared identity, experience and status. This is best demonstrated by Ava's story:

Ava: $\quad$ The people I go out with there is always something stupid or funny that happens. When we were in Amsterdam we were in this club. We were all drunk and literally for some strange reason we were all talking to each other and this like weird sort of cloud - like gas/cold air went shhhh down and we were all talking and it went in our mouths and we couldn't see anything and it was like all in our mouths. And it's just like weird and it makes weird memories doesn't it. I have never ever experienced anything like that before in my life and I was like why are they choking people?

Researcher: So that becomes a talking point for afterwards does it?

Ava: $\quad$..And there are a million other stupid stories that happen as well. Its good isn't it - I like that. 
Researcher: So it's about the retelling as well as the actual night?

Ava: $\quad$ Yes

As noted above reconstructing the drinking experience through storytelling was a key practice. What is relevant, as Ava explains, is that there needs to be something that differentiates the PNO event from the sobriety of the everyday to give the storytelling credibility. In the example above for instance would the same reaction have happened had Ava and her friends not been intoxicated, would it have been less amusing and more scary? These type of incidents are not just 'things' or 'actions' that happened but are what makes an intoxicated experience memorable and even gives it a mythical status. The stories are told and retold and in this way create shared memories and meaning.

\section{Discussion}

This study is unique in its focus on exploring practices that interconnect with intoxicated drinking occasions. The data demonstrates how a PNO consists of a bundle of six interconnected sequential practices; planning, getting ready, predrinking, going out, getting home and storytelling. Within this distinctive bundle we can see how young people's pharmacological intoxication interconnects to other social practices and how it is bounded and delimited by the various competitive practices that restrict participation. Inequalities of knowledge and material resources, determine which practitioners can define what a proficient and acceptable performance looks like (Watson, 2017). For example, participants 
who had to drink in cheap, shabby places because of a lack of financial resources did not have the power to disrupt the practice competences of appropriate places to frequent.

The data also serves to deepen understandings of young people's intoxicated drinking (see Niland et al., 2013; Fry, 2011) and demonstrate how achieving a successful/acceptable performance of the PNO is related to practice competences of achieving and maintaining an acceptable level of intoxication. That is not to say the young people do not get drunk when participating in any other social practice, rather, that other social practices do not necessarily require drunkenness for competent and meaningful engagement (see Ally et al., 2016). Furthermore, the data demonstrates that there is no single acceptable way of performing the practice: rather performativity relates to localised practice which have been shown in this study to be both classed and gendered.

This analysis adds to our understanding of the impact of technological advances and the affordances of social media on intoxicated drinking occasions. Technology is not only constitutive of the materiality of the bundle arrangement but it also serves to illustrate how young people extend their interaction with both their peer group and their wider social networks through the affordances of social media. The data highlights how the affordances of social media have facilitated the practices of planning, getting ready and storytelling and as such provide an example of how infrastructures namely new mobile technologies interconnect 
with and help to shape this bundle arrangement of alcohol consumption (Shove and Trentmann, 2018).

This data has shown how in the context of the PNO, performance in the virtual world is a ubiquitous part of the drinking occasion and that there are various performances of the PNO for the different audiences including both real-time and virtual online performances. Both the real-time and virtual performances of the bundle provided an opportunity to accrue social status and recognition through a shared understanding of what constitutes a proficient gendered performance, demonstrated through likes, shares and retweets generated from virtual social networks.

Similar to the findings of McCreanor et al. (2013) online performances in the study were an essential part of the practice of 'storytelling' and served to differentiate intoxicated drinking occasions from the sober and more mundane nature of everyday life. However, as the data shows there is not just one singular online performance but multiple performances for the different audiences associated with the various online communities, that the young people belong to. The data also reinforces the conclusions of Atkinson et al. (2015) that online drinking performance are carefully crafted and managed in an attempt to ensure a successful performance. However, the findings suggest this managed online performance is undertaken by both young women and young men although there are gendered differences. The identification of multiple online practice 
performances also has implications for social practice itself, perhaps most importantly recognising that practice-as-performance plays out differently and variously in the multifarious virtual worlds.

\section{Conclusion}

While the argument that the use of practice theories would make a useful contribution to understanding (un)healthy behaviours has been made before, the operationalisation of this theoretical perspective in understanding the alcohol consumption practices of young people outside of a university environment is original.

In conclusion, this study has provided an empirical example of how a practice orientated approach can and does provide a useful mechanism for broadening our understandings of how alcohol consumption practice interconnects with other social practices to provide a hybrid entity that reinforces intoxicated alcohol consumption. From this study it is evident that if we want to change this type of drinking pattern, the links (inter)connecting the elements within each practice and those (inter)connecting the practice bundle would need to be broken or reconfigured in some way. It follows that interventions should not be separated from the wider society in which these alcohol consumption practices take place and that any intervention should be interdisciplinary and aimed at the practice rather than at an individual level. If the intention is to prevent participation in the practice, policy makers could seek to support the development of competing 
practices, which contest both the temporal and financial resources that have been taken up by the practice of a PNO.

\section{Acknowledgments}

This study was funded by Liverpool and Lancaster Universities Collaboration for Public Health Research, part of the NIHR SPHR. Thank you to all the young people who participated in this research project and allowed me the honour of being part of their online lives for the duration of the project.

\section{References}

Ally, A., Lovatt, M., Meier, P., Brennan, A. and Holmes, J. (2016) 'Developing a social practice-based typology of British drinking culture: Implications for alcohol policy analysis', Addiction, 111(9), pp. 1568-1579.

Atkinson, A., Ross, K., Begley, E. and Sumnall, H. (2015) 'Constructing alcohol identities: The role of Social Networking Sites in young people's drinking cultures', Alcohol research UK (Accessed 05/02/2016).

Bailey, L., Griffin, C. and Shankar, A. (2015) 'Not a good look": Impossible Dilemmas for Young Women Negotiating the Culture of Intoxication in the United Kingdom', Substance use \& misuse, 50(6), pp. 747-758.

Banks, M. (2008) Using visual data in qualitative research. London: Sage.

Blue, S., Greene, M. and Morosanu, R. (2014) 'Time and practice', in Foulds, C., Jenson, C.L., Blue, S. and Morosanu, R. (eds.) Practices, the Built 
Environment and Sustainability - A Thinking Note Collection.

Cambridge, Copenhagen, London GSI, DIST, BSA CCSG.

Blue, S., Shove, E., Carmona, C. and Kelly, M. (2016) 'Theories of practice and public health: understanding (un)healthy practices', Critical Public Health, 26(1), pp. 36-50.

Braun, V. and Clarke, V. (2006) 'Using thematic analysis in psychology', Qualitative Research in Psychology, 3(2), pp. 77-101.

Braun, V. and Clarke, V. (2013) Successful qualitative research: a practical guide for beginners. London: Sage.

Campbell, H. (2000) 'The glass phallus: Pub(lic) masculinity and drinking in rural New Zealand', Rural sociology, 65(4), pp. 562-581.

Chatterton, P. and Hollands, R. (2003) Urban nightscapes: Youth cultures, pleasure spaces and corporate power. London: Routledge.

Dempster, S. (2011) 'I drink, therefore I'm man: gender discourses, alcohol and the construction of British undergraduate masculinities', Gender and Education, 23(5), pp. 635-653.

Duff, C. (2018) 'Practising drinking, practising health', Addiction, 113(2), pp. 214-215. 
Forsyth, A. (2010) 'Front, side, and back-loading: Patrons' rationales for consuming alcohol purchased off-premises before, during, or after attending nightclubs', Journal of substance use, 15(1), pp. 31-41.

Fraser, S. (2018) 'Do practice approaches go far enough in shifting focus from the individual?', Addiction, 113(2), pp. 215-216.

Fry, M.-L. (2011) 'Seeking the pleasure zone: Understanding young adult's intoxication culture', Australasian Marketing Journal (AMJ), 19(1), pp. 65-70.

Gram-Hanssen, K. (2011) 'Understanding change and continuity in residential energy consumption', Journal of Consumer Culture, 11(1), pp. 61-78.

Gram-Hanssen, K. (2015) 'Structure and agency in understanding and researching practices', in Foulds, C., Jensen, C.L., Blue, S. and Morosanu, R. (eds.) Practices, the built environment \& sustainability, Responses to the thinking note collection. Cambridge, Copenhagen and London: GSI, DIST, BSA CCSG.

Hargreaves, T. (2011) 'Practice-ing behaviour change: Applying social practice theory to pro-environmental behaviour change', Journal of Consumer Culture, 11(1), pp. 79-99.

Haydock, W. (2014) '20 tins of Stella for a fiver': The making of class through Labour and Coalition government alcohol policy', Capital \& Class, 38(3), pp. 583-600. 
Hitchings, R. (2012) 'People can talk about their practices', Area, 44(1), pp. 6167.

Holman, D. and Borgstrom, E. (2016) 'Applying social theory to understand health-related behaviours', Medical humanities, 42(2), pp. 143-5.

Jenkins, T. (1994) 'Fieldwork and the perception of everyday life', Man, 29(2), pp. 433-455.

Kelly, M. and Barker, M. (2016) 'Why is changing health-related behaviour so difficult?', Public health, 136, pp. 109-116.

Mackiewicz, A. (2015) 'Alcohol, young women's culture and gender hierarchies', in Staddon, P. (ed.) Women and Alcohol: Social Perspectives. Bristol: Policy press, pp. 65.

MacLean, S., Savic, M., Pennay, A., Dwyer, R., Stanesby, O. and Wilkinson, C. (2018) 'Middle-aged same-sex attracted women and the social practice of drinking', Critical Public Health, pp. 1-12.

Maller, C. (2015) 'Understanding health through social practices: performance and materiality in everyday life', Sociology of health \& illness, 37(1), pp. 52-66. Martens, L. (2012) 'Practice 'in talk' and talk 'as practice': Dish washing and the reach of language', Sociological Research Online, 17(3), pp. 22. 
Martens, L., Halkier, B. and Pink, S. (2014) 'Researching habits: Advances in linguistic and embodied research practice', International Journal of Social Research Methodology, 17(1), pp. 1-9.

Martin, K., Nelson, J. and Lynch, S. (2013) 'Effectiveness of school-based lifeskills and alcohol education programmes: A review of the literature', (Accessed 06/04/2014).

McCreanor, T., Lyons, A., Griffin, C., Goodwin, I., Moewaka Barnes, H. and Hutton, F. (2013) 'Youth drinking cultures, social networking and alcohol marketing: Implications for public health', Critical public health, 23(1), pp. 110120.

McCreanor, T., Lyons, A., Moewaka Barnes, H., Hutton, F., Goodwin, I. and Griffin, C. (2015) 'DDrink a 12 box before you go': pre-loading among young people in Aotearoa New Zealand', Kotuitui: New Zealand Journal of Social Sciences Online, 11(1), pp. 36-46.

Measham, F. (2006) 'The new policy mix: Alcohol, harm minimisation, and determined drunkenness in contemporary society', International Journal of Drug Policy, 17(4), pp. 258-268.

Measham, F. and Brain, K. (2005) 'Binge' drinking, British alcohol policy and the new culture of intoxication', Crime, media, culture, 1(3), pp. 262-283. 
Meier, P., Warde, A. and Holmes, J. (2018) 'All drinking is not equal: how a social practice theory lens could enhance public health research on alcohol and other health behaviours', Addiction, 113(2), pp. 206-213.

Nairn, A. and Spotswood, F. (2015) '“'Obviously in the cool group they wear designer things" A social practice theory perspective on children's consumption', European Journal of Marketing, 49(9/10), pp. 1460-1483.

Niland, P., Lyons, A., Goodwin, I. and Hutton, F. (2013) '“Everyone can loosen up and get a bit of a buzz on": Young adults, alcohol and friendship practices', International Journal of Drug Policy, 24(6), pp. 530-537.

Reckwitz, A. (2002) 'Toward a Theory of Social Practices: A development in culturalist theorizing', European journal of social theory, 5(2), pp. 243-263. Roberts, M. (2013) 'A big night out': Young people's drinking, social practice and spatial experience in the 'liminoid' zones of English night-time cities', Urban Studies, 52(3), pp. 571-588.

Schatzki, T. (2012) 'A primer on practices', in Higgs, J., Barnett, J., Billett, S., Hutchings, M. and Trede, F. (eds.) Practice-based education. Rotterdam: Sense publishers, pp. 13-26.

Schatzki, T. (2016) 'Sayings, texts and discursive formations', in Hui, A., Schatzki, T. and Shove, E. (eds.) The Nexus of Practices. London: Routledge, pp. 138-152. 
Schatzki, T., Knorr-Cetina, K. and Von Savigny, E. (2001) The practice turn in contemporary theory. Abingdon: Routledge.

Shove, E., Pantzar, M. and Watson, M. (2012) The dynamics of social practice: everyday life and how it changes. London: Sage.

Shove, E. and Trentmann, F. (2018) Infrastructures in practice: the dynamics of demand in networked societies. Routledge.

Social Research Association (2003) 'Ethical guidelines', (Accessed 23/02/2016).

Spotswood, F., Chatterton, T., Tapp, A. and Williams, D. (2015) 'Analysing cycling as a social practice: An empirical grounding for behaviour change', Transportation research part F: traffic psychology and behaviour, 29, pp. 2233.

Supski, S., Lindsay, J. and Tanner, C. (2017) 'University students' drinking as a social practice and the challenge for public health', Critical Public Health, 27(2), pp. 228-237.

Thom, B., Herring, R., Thickett, A. and Duke, K. (2016) 'The Alcohol Health Alliance: The emergence of an advocacy coalition to stimulate policy change', British Politics, 11(3), pp. 301-323.

Thurnell-Read, T. (2013) 'Yobs' and 'Snobs': Embodying Drink and the Problematic Male Drinking Body', Sociological Research Online, 18(2), pp. 3. 
Twine, R. (2015) 'Understanding snacking through a practice theory lens', Sociology of Health \& Illness, 37(8), pp. 1270-1284.

Waitt, G. and De Jong, A. (2014) 'Embodied geographies of alcohol and the weekend in the Bega Valley, New South Wales, Australia', Leisure Studies, 33(2), pp. 116-132.

Warde, A. (2005) 'Consumption and theories of practice', Journal of consumer culture, 5(2), pp. 131-153.

WHO (2012) Action Plan for implementation of the European Strategy for the Prevention and Control of Noncommunicable Diseases 2012-2016,

Copenhagen: World Health Organization. Available at:

http://www.euro.who.int/ data/assets/pdf file/0003/147729/wd12E NCDs 11

1360 revision.pdf?ua=1 (Accessed: 13/07/2016).

WHO (2018) Global status report on alcohol and health 2018, Geneva: WHO. Available at:

https://www.who.int/substance_abuse/publications/global_alcohol_report/en/ (Accessed: 22/10/2018). 